BIO Web of Conferences 2, 01003 (2014)

DOI: $10.1051 /$ bioconf/ 20140201003

C Owned by the authors, published by EDP Sciences, 2014

\title{
Formation of telluric planets and the origin of terrestrial water
}

\author{
Sean Raymond ${ }^{1, a}$ \\ ${ }^{1}$ Laboratoire d'Astrophysique de Bordeaux, UMR 5804
}

\begin{abstract}
Simulations of planet formation have failed to reproduce Mars' small mass (compared with Earth) for 20 years. Here I will present a solution to the Mars problem that invokes large-scale migration of Jupiter and Saturn while they were still embedded in the gaseous protoplanetary disk. Jupiter first migrated inward, then "tacked" and migrated back outward when Saturn caught up to it and became trapped in resonance. If this tack occurred when Jupiter was at 1.5 AU then the inner disk of rocky planetesimals and embryos is truncated and the masses and orbits of all four terrestrial planet are quantitatively reproduced. As the giant planets migrate back outward they re-populate the asteroid belt from two different source populations, matching the structure of the current belt. C-type material is also scattered inward to the terrestrial planet-forming zone, delivering about the right amount of water to Earth on 10-50 Myr timescales.
\end{abstract}

\section{Introduction}

\section{The formation of terrestrial planets}

As described in the Introduction, the final stages of terrestrial planet formation take place in the presence of any giant planets that may have formed. In addition, it is during this phase that bodies are large enough that during gravitational close encounters they can obtain large eccentricities. Thus, the feeding zones of the terrestrial planets are determined during their final accretion. The composition of each planet is simply a massweighted combination of the initial composition of all the material in its feeding zone.

The inner Solar System is thought to have been too hot to allow water to condense [1], [2]. Thus, it is widely thought that Earth's local building blocks were dry and that water was "delivered" via impacts from objects that condensed at cooler temperatures. Earth's water is a chemical match to carbonaceous chondrite meteorites (fragments of C-type asteroids) in terms of $\mathrm{D} / \mathrm{H}$ and other isotopic ratios [3], [4].

Simulations like the one illustrated in figure 2 above have the attractive feature of explaining the origin of Earth's water [6], [7], [5], [8]. In such simulations the feeding zones of the terrestrial planets spread outward and widen in time such that a fraction of the Earth's component mass originated in the outer asteroid belt, beyond $3 \mathrm{AU}$. The material that originated in that cold location thus represents the source of water on Earth in that model. This water-rich material slowly diffuses inward during

ae-mail: sean.raymond@obs.u-bordeaux1.fr 


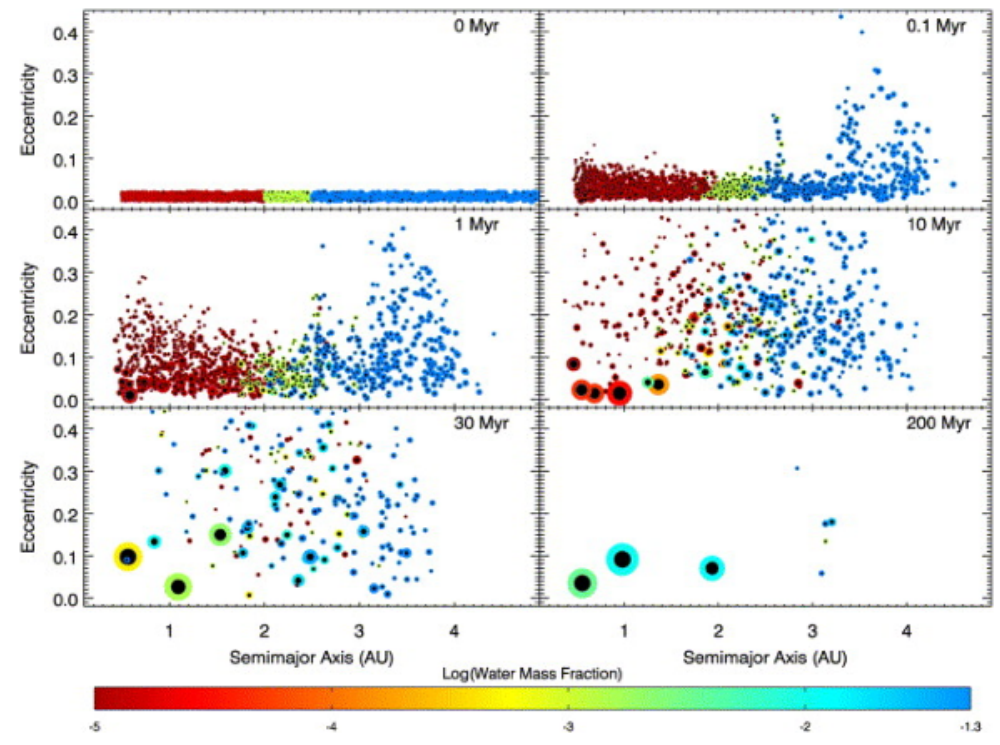

Figure 1. Six snapshots in time from simulation 0 , with 1885 initial particles. The size of each body corresponds to its relative physical size (i.e., its mass $\mathrm{M} 1 / 3 \mathrm{M} 1 / 3$ ), but is not to scale on the $\mathrm{x}$ axis. The color of each particle represents its water content, and the dark inner circle represents the relative size of its iron core. There is a Jupiter-mass planet at 5.5 AU on a circular orbit (not shown). From [5].

the late stages of accretion by multiple gravitational scattering events, and finds its way into the inner Solar System.

It has been showed that the standard model of terrestrial planet formation cannot reproduce the Solar System's terrestrial planets [5], [9]. Rather, as shown in figure 2, simulations invariably form a "Mars" that is far larger than the real one, and often strand Mars-sized embryos in the asteroid belt. Note that in this context I refer to the "standard" model as one in which the giant planets were on or near their current orbits at the time of terrestrial planet formation.

There are certain situations in which the standard model of terrestrial planet formation can produce a small Mars. If, during accretion, Jupiter and Saturn were at their current orbital radii but had modestly higher orbital eccentricities of $0.075-0.1$ each, then the nu6 secular resonance located at 2.1 AU would have been much stronger. This resonance acts to quickly pump the eccentricities of nearby particles to such high values that they either interact with an embryo in the inner disk or fall into the Sun [10]. In such a configuration the nu6 resonance can efficiently clear out a large fraction of the mass in Mars' nominal feeding zone and produce a small Mars [11], [9]. However, this scenario is not consistent with the evolution of the Solar System because it requires that Jupiter and Saturn reached their final orbital radii on a shorter timescale than Mars' known accretion timescale of 3-5 Myr [12]. Migration and/or scattering among the ice giants is required to reproduce the observed structure of the Kuiper belt [13], [14]. This migration cannot have dynamically occurred on such a short timescale (e.g., [15]), and so we rule this scenario out. 


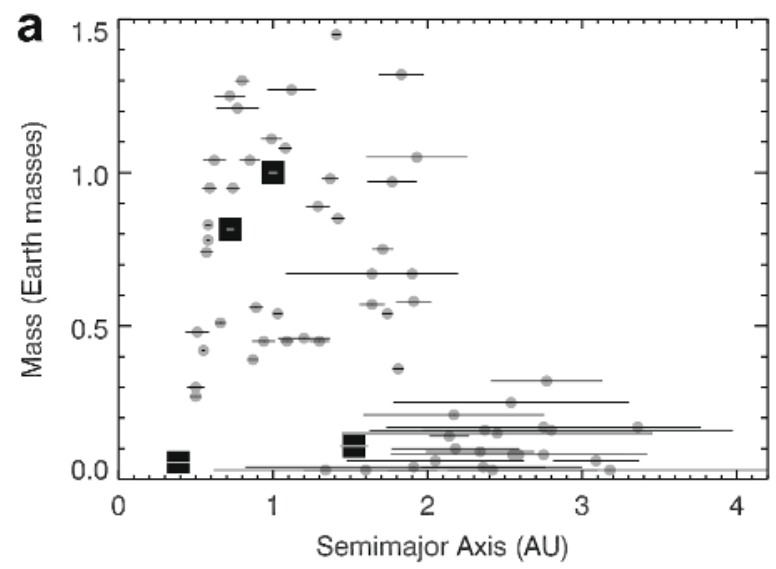

Figure 2. Mass vs orbital radius for the real terrestrial planets (black squares) and simulated ones (grey dots) from the "standard model" described in the text. Although Earth and Venus are modestly-well reproduced, Mars "analogs" are far too massive. From [9].

To develop a more consistent model, we needed to return to the orbital evolution of Jupiter and Saturn at early times while they were still embedded in the gaseous protoplanetary disk. Alone in the disk, Jupiter's core should have grown, type 1 migrated towards a convergence zone, and started to accrete gas from the disk. Once its mass reached a critical threshold it should have undergone rapid gas accretion, cleared out a gap in the disk and type 2 migrated inward until the disk dissipated. But Jupiter was not alone in the disk. Saturn's presence changes the picture considerably at later times. Since it is smaller and more distant, Saturn probably accreted more slowly than Jupiter. When Jupiter became a gas giant and started migrating inward, Saturn was still a core slowly accreting gas. Saturn should have followed the same basic evolution as Jupiter: accreted gas slowly, then undergone rapid runaway gas accretion and type 2 migrated inward toward Jupiter. Simulations show that, when this happens, Saturn actually migrates inward much faster than Jupiter because there is a small mass window in which migration is especially fast, sometimes called type 3 [16]. The relevant mass range is for planets at about the gap-clearing mass, located from 50-200 Earth masses.

Saturn type 3 migrated inward very quickly and became stabilized when it was trapped in resonance with Jupiter. Hydrodynamical simulations consistently show that the two planets are typically trapped in 3:2 mean motion resonance [17]. When in this configuration, a fascinating hydrodynamical effect takes place whereby the two giant planets share a common gap that is deeper in its inner parts (because Jupiter is more massive). Saturn is able to "push" gas across the gap onto the inner disk, thereby decoupling the evolution of the planets from the inward viscous flow of the disk. The gravitational back-reaction of the pushing of this gas inward essentially pushes the planets outward [18]. Thus, instead of type 2 migrating inward as a single planet would, the Jupiter-Saturn system naturally migrates outward [19], [20]. This has been confirmed with detailed hydrodynamical calculations by several groups such as the one shown in figure 3 .

To summarize, we have shown that Jupiter and Saturn's orbits were not static after their formation. While the gaseous disk was still present, their natural evolution was a twophased, 

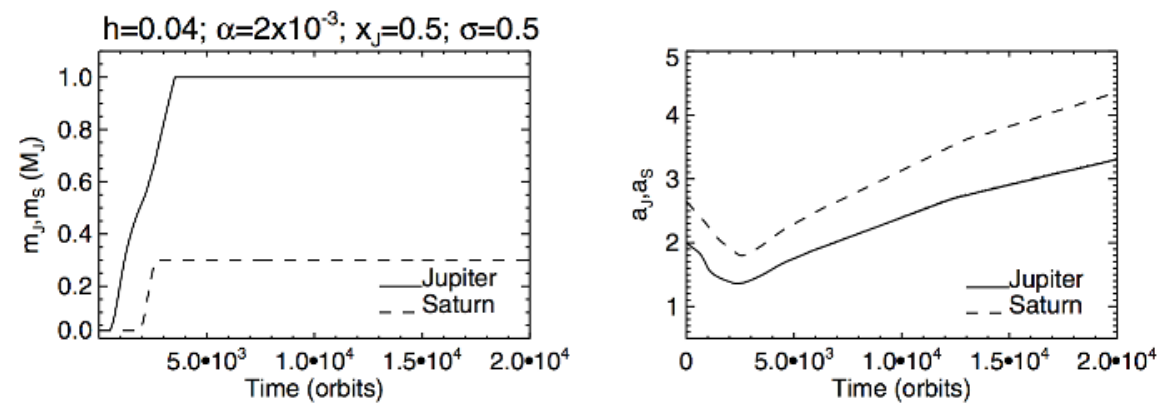

Figure 3. A hydrodynamical simulation of Jupiter and Saturn embedded in the gaseous protoplanetary disk. Jupiter and Saturn both migrate inward as they grow but once the appropriate conditions are met - they are trapped in resonance with sufficiently large masses and a mass ratio between about $0.25-0.5$ ([18]) - the planets migrate outward to large orbital radius. From [21].

inward-then-outward migration [21]. The distance of inward migration was controlled by the speed of Jupiter's inward migration and the time lapsed between its formation and Saturn's. The distance of outward migration is determined by the lifetime of the disk; as the mass in gas waned the rate of migration slowed and eventually stopped. Below a critical disk mass threshold (a few to $10 \%$ of the minimal value) the likelihood of long-range outward migration is small [22]. The turnaround point is completely unconstrained by this theory.

Let us return to terrestrial planet formation. Recall that the standard model - with static orbits of Jupiter and Saturn - inevitably produces Mars analogs that are much too large. This problem can be solved if the initial conditions for terrestrial accretion were much different than those assumed in most simulations, i.e., a continuous disk of planetary embryos and planetesimals extending from an inner edge ( 0.3-0.5 AU) out to Jupiter's present-day orbit (e.g., [5], [9]). If, instead of a continuous disk, the terrestrial planets formed from a narrow annulus of material with an outer edge at about $1 \mathrm{AU}$ then a small Mars forms naturally [23], [24]. In this context Mars is an "edge effect", an embryo scattered out of the dense annulus into the sparselypopulated ambient region. Mars' growth was stunted simply by running out of building blocks once it left the dense annulus. Likewise, in this model Mercury was scattered interior to the inner edge of the annulus.

The annulus model for terrestrial planet formation has the advantage of reproducing a lot of characteristics of the terrestrial planets: masses, orbits, and formation timescales. And best of all, it naturally forms a small Mars that is compatible with all observational constraints. However, the idea is completely ad hoc and has no theoretical or observational backing. No sharp edge is thought to have existed in the gas' radial density distribution; small discontinuities likely existed due to transition in viscosity regimes (e.g., [25]) but these are too narrow to explain Mars' small size. What if embryos only formed out to $1 \mathrm{AU}$ but there were only planetesimals farther out? In that case the embryo swarm would simply spread out due to "orbital repulsion" and occupy the entire available realm [26]. What if planetesimals only formed out to 1 AU and there was only dust farther out? In that case the planetesimal swarm would spread out to fill the available space, much like the previous case [27]. 
What if Jupiter were at 1.5 AU? In that case, any embryos or planetesimals exterior to $\sim 1 \mathrm{AU}$ would be dynamically ejected very quickly and there would indeed be the sharp edge needed to reproduce Mars.

\section{The "Grand Tack" model}

In the context of the inward-then-outward migration of Jupiter and Saturn described above, there was no constraint on the turnaround point, where Jupiter "tacked" (a sailing term for a change of direction against the wind) and started migrating outward. What if that turnaround point was at 1.5 AU? This idea represented the birth of the "Grand Tack" model.

In the Grand Tack model, Jupiter migrates across the asteroid belt twice. Given that Jupiter is about 300,000 times more massive than the entire asteroid belt, how do we have an asteroid belt at all? The answer is shown in figure 4. As Jupiter migrated inward, it both shepherded planetesimals inward via its interior 2:1 and 3:2 resonances (by the mechanism described in Raymond et al 2006, Science) and scattered planetesimals outward onto more distant eccentric orbits. About $10 \%$ of the planetesimals initially interior to Jupiter survived on eccentric scattered orbits. Next, when Jupiter and Saturn migrated back outward, they encountered both the scattered planetesimals (red in figure 4) and local primitive planetesimals (light and dark blue in Fig 9). During the giant planets' migration, any planetesimal that crossed their path was scattered onto an eccentric orbit and in most cases ejected from the Solar System. The process of ejection requires several close gravitational encounters and many planetesimals spend time on orbits interior to Jupiter's during this phase (although their orbits still cross Jupiter's of course). In the simplified gravitational problem the planetesimals must reencounter the giant planets until they are ejected. However, given that the giant planets are migrating outward, a fraction of inwardscattered planetesimals are stranded as Jupiter migrates out of the way. These planetesimals thus survive on scattered orbits interior to the giant planets, whose gravitational influence diminishes as they become more and more separated from the asteroid belt. Although the efficiency of this process is only $\sim 1 \%$, it is entirely sufficient to populate the asteroid belt with several times its current mass, which is needed given that the belt is later depleted by chaotic diffusion [29] and by late heavy bombardment [30].

The Grand Tack also explains why the inner asteroid belt is dominated by generally igneous S-types and the outer belt by more "primitive" C-types. As the giant planets migrated outward, they encountered the planetesimals that had been scattered outward during inward migration (the red dots) before the primordial outer planetesimals (the blue dots). After inward-scattering a planetesimal's final orbit is correlated with Jupiter's orbit at the time of last scattering, since the planetesimal's orbit crossed Jupiter's. Thus, the earliest-scattered bodies were implanted closer-in than the later-scattered planetesimals. If we assume the initially inner planetesimals are S-types and initially outer planetesimals are C-types, then the post-giant planet migration asteroid belt does indeed contain a radial dichotomy very similar to the current-day belt, with S-types dominating the inner belt and C-types the outer belt.

The Grand Tack model naturally reproduces the orbital and mass distribution of the terrestrial planets. The model can also explain the origin of Earth's water. In the standard model, Earth's feeding zone extended outward such that a fraction of its building blocks were water-rich [6], [7], [8]. In the Grand Tack model the terrestrial planets form from a narrow annulus of embryos and planetesimals that was sculpted by Jupiter. The terrestrial planets should be dry. However, this annulus was contaminated during the giant planets' outward migration. As can be seen in figure 4, 


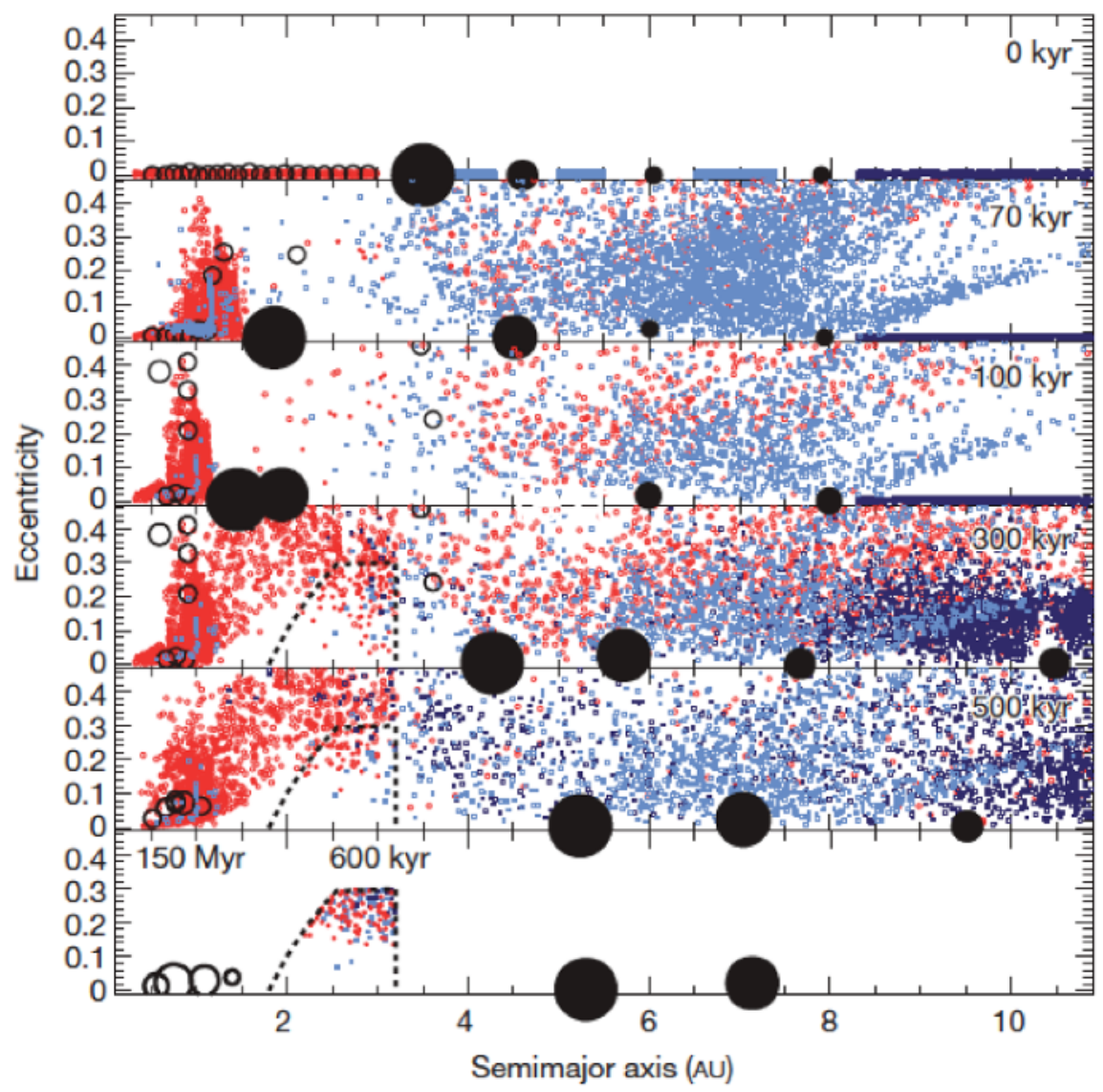

Figure 4. A representative simulation of the Grand Tack model [28]. Here the black dots represent the giant planets. Jupiter and Saturn follow the evolution described in the text. The red dots are planetesimals that originated interior to Jupiter's orbit, the light blue dots planetesimals starting between the giant planets, and the dark blue dots more distant planetesimals. The open circles are terrestrial embryos. After the giant planets' two-phase migration the whole inner Solar System is reproduced: the terrestrial planets' masses and orbits and the mass, mass distribution and radial dichotomy of the asteroid belt.

many planetesimals that were scattered inward by Jupiter "overshot" the asteroid belt and ended up on orbits that entered the inner Solar System. Indeed, for every planetesimal that was trapped on a stable orbit in the asteroid belt, 10-20 were scattered onto orbits with perihelion distances of 1-1.5 AU. These scattered C-type bodies were sufficient in number to deliver the requisite amount of water to Earth (see figure 5). Compared with the standard model, a factor a few less water is delivered to Earth, but still several times more than its current water budget [31]. And this water also matches the chemical constraints because it is also delivered by C-types, although in this context by the same parent population that was implanted into the asteroid belt as Ctypes. 


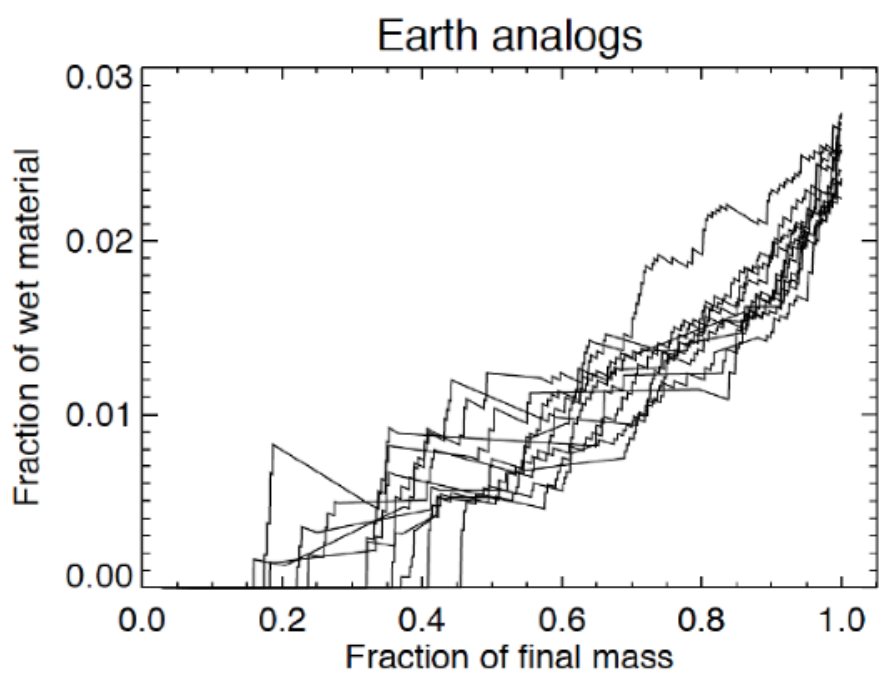

Figure 5. Water delivery to Earth analogs in the Grand Tack model (from O'Brien et al, in preparation). The plot shows the fraction of wet (C-type) material accreted by each simulated Earth-like planet throughout its growth.

Jupiter and Saturn's outward migration may also have stimulated a late phase of accretion of the ice giants. figure 6 shows a simulation of the last phases of the Grand Tack model with Jupiter and Saturn's outward migration slowing down in a dissipating disk. Uranus and Neptune start as 5 Earth mass cores embedded in a swarm of $100 \mathrm{~m}$ planetesimals. The orbital compression caused by the inward-migrating cores and the outward-migrating giant planets creates a very rapid phase of growth and the ice giants reach nearly their final masses on a 100,000 year timescale. The growth itself is catalyzed by close encounters between the ice giants, which scatter each other into pristine, very dynamically cold rings of planetesimals that are efficiently accreted (Raymond et al, in preparation).

In the example from figure 6 the planetesimals were extremely small and thus any eccentricities or inclinations were efficiently damped by gas drag with the disk [32]. The ice giants' growth is much more limited if the planetesimals were larger. However, the collisional cross-section of Earth-mass or larger cores should naturally be enhanced if they had tenuous gaseous envelopes [33]. Depending on the properties of the planet/disk and the size of the planetesimals, the effective accretion cross section could be increased by a factor of a few to 100 . When taking this effect into account the size-dependence of ice giant accretion is removed. The current-day ice giants' masses become relatively easy to reproduce as long as the initial cores were at least 1 Earth mass in size and there was sufficient mass in the planetesimal swarm.

\section{References}

[1] A.P. Boss, Annual Review of Earth and Planetary Sciences 26, 53 (1998) 
BIO Web of Conferences

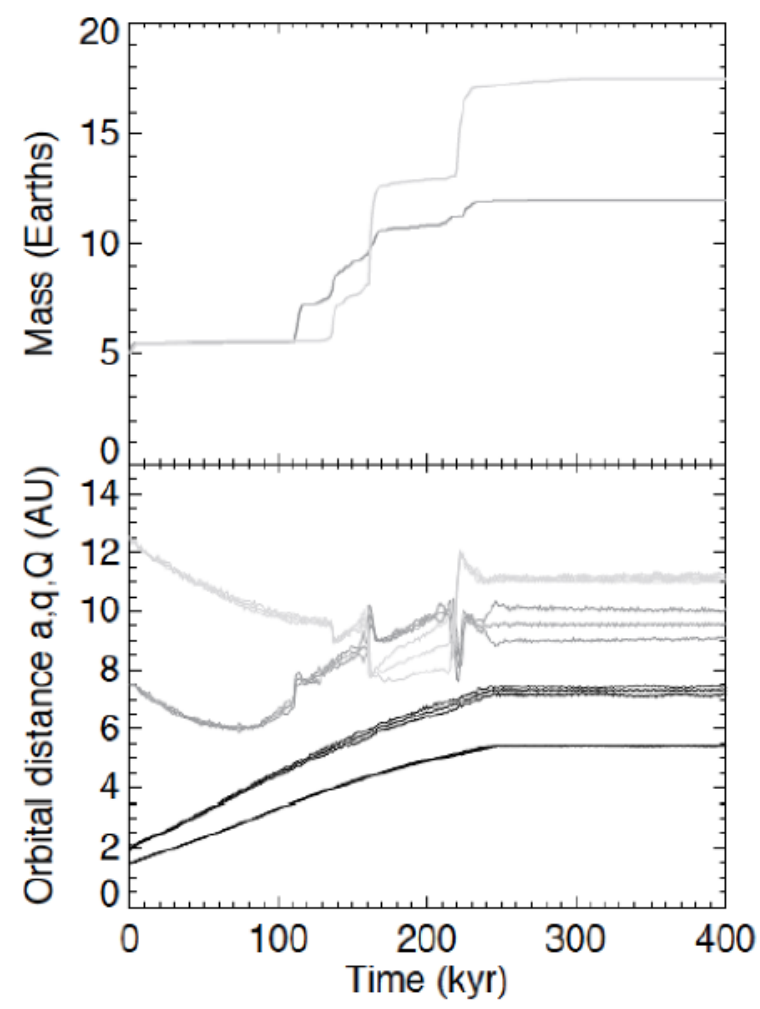

Figure 6. Accretion of Uranus and Neptune during Jupiter and Saturn's outward migration, in this case starting from two 5 Earth mass embryos embedded in a swarm of small $(100 \mathrm{~m})$ planetesimals. From Raymond et al (2013, in preparation).

[2] M. Podolak, The Location of the Snow Line in Protostellar Disks, in IAU Symposium, edited by J.A. Fernandez, D. Lazzaro, D. Prialnik, R. Schulz (2010), Vol. 263 of IAU Symposium, pp. $19-28,0911.4803$

[3] J.F. Kerridge, Geoch. \& Cosmoch. Acta. 49, 1707 (1985)

[4] B. Marty, R. Yokochi, J.M. Schaefer, R. Wieler, Geochimica et Cosmochimica Acta Supplement 70, 396 (2006)

[5] S.N. Raymond, T. Quinn, J.I. Lunine, Icarus 183, 265 (2006), arXiv : astro-ph/0510284

[6] A. Morbidelli, J. Chambers, J.I. Lunine, J.M. Petit, F. Robert, G.B. Valsecchi, K.E. Cyr, Meteoritics and Planetary Science 35, 1309 (2000)

[7] S.N. Raymond, T. Quinn, J.I. Lunine, Icarus 168, 1 (2004), arXiv: astro-ph/0308159

[8] S.N. Raymond, T. Quinn, J.I. Lunine, Astrobiology 7, 66 (2007), arXiv: astro-ph/0510285

[9] S.N. Raymond, D.P. O'Brien, A. Morbidelli, N.A. Kaib, Icarus 203, 644 (2009), 0905 . 3750

[10] B.J. Gladman, F. Migliorini, A. Morbidelli, V. Zappala, P. Michel, A. Cellino, C. Froeschle, H.F. Levison, M. Bailey, M. Duncan, Science 277, 197 (1997)

[11] E. Thommes, M. Nagasawa, D.N.C. Lin, ApJ. 676, 728 (2008), 0802.0541

[12] N. Dauphas, A. Pourmand, Nature 473, 489 (2011) 
[13] R. Malhotra, AJ 110, 420 (1995), arXiv: astro-ph/9504036

[14] H.F. Levison, A. Morbidelli, C. Van Laerhoven, R. Gomes, K. Tsiganis, Icarus 196, 258 (2008), 0712.0553

[15] J.M. Hahn, R. Malhotra, AJ 117, 3041 (1999), arXiv: astro-ph/9902370

[16] F.S. Masset, J.C.B. Papaloizou, ApJ 588, 494 (2003), arXiv: astro-ph/0301171

[17] A. Pierens, R.P. Nelson, A\&A 482, 333 (2008), 0802.2033

[18] F. Masset, M. Snellgrove, MNRAS 320, L55 (2001), arXiv : astro-ph/0003421

[19] A. Morbidelli, A. Crida, Icarus 191, 158 (2007)

[20] A. Crida, F. Masset, A. Morbidelli, ApJ 705, L148 (2009), 0910 . 1004

[21] A. Pierens, S.N. Raymond, A\&A 533, A131 (2011), 1107.5656

[22] G. D’Angelo, F. Marzari, ApJ 757, 50 (2012), 1207 . 2737

[23] G.W. Wetherill, Accumulation of the terrestrial planets, in IAU Colloq. 52: Protostars and Planets, edited by T. Gehrels (1978), pp. 565-598

[24] B.M.S. Hansen, ApJ 703, 1131 (2009), 0908.0743

[25] L. Jin, W.D. Arnett, N. Sui, X. Wang, ApJ 674, L105 (2008)

[26] E. Kokubo, S. Ida, Icarus 123, 180 (1996)

[27] Z.M. Leinhardt, D.C. Richardson, G. Lufkin, J. Haseltine, MNRAS 396, 718 (2009), 0903.2354

[28] K.J. Walsh, A. Morbidelli, S.N. Raymond, D.P. O’Brien, A.M. Mandell, Nature 475, 206 (2011), 1201.5177

[29] D.A. Minton, R. Malhotra, Icarus 207, 744 (2010), 0909. 3875

[30] A. Morbidelli, R. Brasser, R. Gomes, H.F. Levison, K. Tsiganis, AJ 140, 1391 (2010), 1009.1521

[31] B. Marty, Earth and Planetary Science Letters 313, 56 (2012)

[32] I. Adachi, C. Hayashi, K. Nakazawa, Progress of Theoretical Physics 56, 1756 (1976)

[33] S. Inaba, M. Ikoma, A\&A 410, 711 (2003) 
